\title{
A-DROP and Blood Urea Nitrogen-to-Serum Albumin Ratio are Useful in Assessing the Severity of Pneumocystis Pneumonia in Patients Without Human Immunodeficiency Virus Infection: A Retrospective Study
}

Jumpei Akahane

Shinshu University School of Medicine https://orcid.org/0000-0001-8648-6547

Atsuhito Ushiki ( $\square$ atsuhito@shinshu-u.ac.jp )

Shinshu University School of Medicine https://orcid.org/0000-0001-8158-6100

Makoto Kosaka

Nagano Prefectural Shinshu Medical Center

Yuichi lkuyama

Shinshu University School of Medicine

Akemi Matsuo

Minaminagano Medical Center Shinonoi General Hospital

Tsutomu Hachiya

Japanese Red Cross Society Suwa Hospital

Fumiaki Yoshiike

Nagano Municipal Hospital

Shigeru Koyama

Japanese Red Cross Society Nagano Hospital

Masayuki Hanaoka

Shinshu University School of Medicine

Research article

Keywords: Pneumocystis pneumonia, Prognostic factors, A-DROP, Blood urea nitrogen-to-serum albumin ratio

Posted Date: August 28th, 2020

DOI: https://doi.org/10.21203/rs.3.rs-52173/v1 
License: (c) (i) This work is licensed under a Creative Commons Attribution 4.0 International License. Read Full License 


\section{Abstract}

Background: There is an increasing incidence of Pneumocystis pneumonia among individuals without the human immunodeficiency virus (HIV) infection (non-HIV Pneumocystis pneumonia). However, the prognostic factors for patients with non-HIV Pneumocystis pneumonia have not been identified. Moreover, A-DROP (for classifying the severity of community-acquired pneumonia) or the blood urea nitrogen-to-serum albumin ratio, which is reported to be predictor of mortality of community-acquired pneumonia, has not been established as an efficient prognostic factor in patients with non-HIV Pneumocystis pneumonia. In this study, we analyzed the prognostic factors for non-HIV Pneumocystis pneumonia and evaluated the effectiveness of A-DROP and the blood urea nitrogen-to-serum albumin ratio as prognostic factors.

Methods: This retrospective study involved a chart review of the medical records of 102 patients diagnosed with non-HIV Pneumocystis pneumonia between January 2003 and May 2019 at five medical facilities. Prognostic factors associated with the 30-day mortality were assessed using multiple logistic regression analysis.

Results: Among the 102 patients with non-HIV Pneumocystis pneumonia, 46 (45.1\%) had autoimmune diseases, 19 (18.6\%) had hematological malignancies, 18 (17.7\%) had solid malignancies, and 19 (18.6\%) had other diseases. The 30-day mortality rate for non-HIV Pneumocystis pneumonia was 20.5\% in this study population. Compared with survivors, non-survivors had significantly lower serum albumin levels and a significantly higher age, corticosteroid dosage at the onset of Pneumocystis pneumonia, alveolar-arterial oxygen gradient, A-DROP score, lactate dehydrogenase levels, blood urea nitrogen levels, and blood urea nitrogen-to-serum albumin ratio. The results of multivariate analysis showed that a high A-DROP score and blood urea nitrogen-to-serum albumin ratio at treatment initiation were significantly associated with the 30-day mortality risk.

Conclusions: A high A-DROP score and blood urea nitrogen-to-serum albumin ratio at treatment initiation are independent prognostic predictors of mortality risk in patients with non-HIV Pneumocystis pneumonia.

\section{Background}

Pneumocystis pneumonia (PcP) is one of the most serious opportunistic infections associated with human immunodeficiency virus (HIV) infection. The introduction of PcP prophylaxis and highly active antiretroviral therapy has reduced the incidence and mortality rates of PcP in HIV-infected patients (HIV PcP) [1, 2]. In contrast, the incidence of PcP in patients without HIV infection (non-HIV PcP) but with malignancies, those who have undergone organ transplantation, and in those undergoing immunosuppressive therapy is increasing [2-4]. The mortality rate was $10-20 \%$ in patients with HIV PcP and $19.6-60 \%$ in patients with non-HIV PcP $[5,6]$. The prognostic factors of non-HIV PcP have not yet been determined; however, several factors, such as high levels of alveolar-arterial oxygen gradient (A- 
$\mathrm{aDO}_{2}$ ), high lactate dehydrogenase (LDH) levels, high blood urea nitrogen (BUN) levels, low serum albumin (Alb) levels, and high pneumonia severity index (PSI), were reported to be associated with a poor prognosis of non-HIV PcP [7-10]. PSI is a scoring system proposed by the American Thoracic Society/Infectious Diseases Society of America for classifying patients with community-acquired pneumonia (CAP) [11]. Alternatively, A-DROP (age, dehydration, respiratory failure, orientation, systolic blood pressure) is a scoring system proposed by the Japanese Respiratory Society (JRS) [12] and used widely for classifying the severity of patients with CAP in Japan. High BUN and low serum Alb levels are considered as poor prognostic factors in patients with CAP [13-16]. In addition, a high BUN-to-serum Alb (BUN/Alb) ratio is reportedly a useful marker of mortality from CAP, hospital-acquired pneumonia (HAP), and aspiration pneumonia [17-19]. A previous report suggested that A-DROP may underestimate the severity of non-HIV PCP [20]. Therefore, A-DROP has not been established as a prognostic factor in patients with non-HIV PcP. The effectiveness of the BUN/Alb ratio also has not yet been evaluated in patients with non-HIV PcP. In this study, we aimed to identify the prognostic factors for patients with nonHIV PcP. We also evaluated the effectiveness of A-DROP and the BUN/Alb ratio, which are useful in patients with CAP, as predictors of prognosis in patients with non-HIV PCP.

\section{Methods}

\section{Study design and population}

A retrospective study was conducted in patients diagnosed with non-HIV PcP between January 2003 and May 2019 at five medical facilities (Shinshu University Hospital, Nagano Municipal Hospital, Minaminagano Medical Center Shinonoi General Hospital, Japanese Red Cross Society Nagano Hospital, and Japanese Red Cross Society Suwa Hospital) in Nagano Prefecture, Japan, on the basis of the data available in their medical records. According to data from previous studies [21], a diagnosis of non-HIV PcP was made in our study if the patients met the following 4 criteria: (i) immunosuppressive status; (ii) diffuse bilateral ground-glass opacity on chest radiography or computed tomography scans; (iii) detection of Pneumocystis jirovecii in respiratory specimens by direct staining or polymerase chain reaction assay; and (iv) elevated plasma $(1 \rightarrow 3)-\beta$-D-glucan levels. The levels of plasma $(1 \rightarrow 3)$ - $\beta$-D-glucan were measured using a Wako $\beta$-glucan test (Wako Pure Chemical Industries, Osaka, Japan) or a Fungitec G test MK (Seikagaku Corporation, Tokyo, Japan). Infection with PcP was indicated if the plasma $(1 \rightarrow 3)$ $\beta$-D-glucan level was over the upper limit of the normal range. The normal range of plasma $(1 \rightarrow 3)-\beta-D-$ glucan level is $\leq 11 \mathrm{pg} / \mathrm{mL}$ in the Wako $\beta$-glucan test and $\leq 20 \mathrm{pg} / \mathrm{mL}$ in the Fungitec $\mathrm{G}$ test MK. Immunosuppressive status was defined as reduced immune status and was seen in patients who suffered from malignancies (hematological or solid), in patients who were treated with immunosuppressive therapy, or in patients who had undergone an organ transplant and hematopoietic stem cell transplant.

\section{Data collection}


The following demographic and clinical data were collected from the medical records of the patients diagnosed with non-HIV PcP: age, gender, body mass index, underlying diseases, treatments for underlying diseases, corticosteroid dosage at the onset of PcP for underlying diseases, PcP prophylaxis, days from symptom appearance (onset of $\mathrm{PcP}$ ) to treatment, physical findings at treatment initiation (percutaneous oxygen saturation $\left(\mathrm{SpO}_{2}\right)$, blood pressure, and status of consciousness), respiratory support (invasive positive-pressure ventilation [IPPV]), initial treatment for PcP, adjunctive corticosteroid therapy, A-DROP score, laboratory findings before treatment, and final outcome. Corticosteroid dosage was expressed as the equivalent dose of prednisolone. Respiratory failure was defined as partial pressure of arterial oxygen $\left(\mathrm{PaO}_{2}\right) \leq 60$ Torr or $\mathrm{SpO}_{2} \leq 90 \%$ in room air. A-DROP is a scoring system proposed by the JRS to assess the severity of CAP [12]. A-DROP was evaluated using the following parameters: (i) age (men $\geq 70$ years, women $\geq 75$ years); ( ()$B U N \geq 21 \mathrm{mg} / \mathrm{dL}$ or dehydration; ( $($ ) respiratory failure; ( () disturbance of consciousness; ( $($ ) hypotension (systolic blood pressure $\leq 90 \mathrm{mmHg}$ ). Each parameter corresponds to 1 point in the A-DROP score. An A-DROP score of 0 is considered mild; $1-2$, moderate; 3 , severe; and 4-5, extremely severe [12]. Consciousness status was assessed using the Glasgow Coma Scale (GCS), and disturbance of consciousness was defined as GCS $\leq 14$. The endpoint of this study was all-cause mortality in the patients with non-HIV PcP within 30 days from treatment initiation.

\section{Statistical analysis}

Data are expressed as proportions for categorical variables and as median (range) for continuous variables. Categorical variables were compared using the chi-squared or Fisher's exact test, and continuous variables were compared using the Mann-Whitney test between the survivors and nonsurvivors. The independence of each variable was tested using Spearman's rank correlation coefficient. Prognostic factors in relation to 30-day mortality were assessed by multiple logistic regression analysis. The receiver-operating characteristic (ROC) curve was used for evaluating the ability of prognostic factors to estimate 30 -day mortality. $\mathrm{P}<0.05$ was considered statistically significant. All statistical analyses were performed using IBM SPSS Statistics software, version 26 (IBM, Armonk, NY, USA).

\section{Results}

\section{Baseline clinical characteristics of patients with non-HIV PcP}

During the investigation period, 102 patients (55 men [53.9\%] and 47 women [46.1\%]) met the diagnostic criteria for non-HIV PcP, and the baseline clinical characteristics of the patients are shown in Table 1. The median age of the patients was 69.5 years (range 22-88 years). Forty-six patients (45.1\%) had autoimmune diseases, 19 (18.6\%) had hematological malignancies, $18(17.7 \%)$ had solid malignancies, and $19(18.6 \%)$ had other diseases. The autoimmune diseases seen in this patient population included rheumatoid arthritis, systemic lupus erythematosus, polymyositis/dermatomyositis, anti-neutrophil cytoplasmic antibody-associated vasculitis, spondyloarthritis, and Goodpasture's syndrome. 
Rheumatoid arthritis was the most common autoimmune disease in the present study, seen in 29 of the 46 patients $(63.0 \%)$ with autoimmune disease. In the entire cohort, regarding treatment modalities for the underlying diseases, 34 patients (33.3\%) were treated with corticosteroids alone and 53 patients $(53.0 \%)$ were treated with corticosteroids in combination with other drugs, such as immunosuppressants, biological agents, and antitumor drugs. Fifteen patients (14.7\%) were treated with therapies other than corticosteroids. Corticosteroids were administered in 87 patients $(85.3 \%)$ at the onset of PcP for the already existing diseases, and the median corticosteroid dosage (prednisolone equivalent) was $15 \mathrm{mg} /$ day (range 2-400 mg/day). Two patients received PcP prophylaxis with trimethoprim/sulfamethoxazole (TMP/SMX).

Patient prognosis was evaluated with 30-day mortality, defined as the mortality at day 30 after initiation of the treatment. The 30-day mortality rate in non-HIV PcP was found to be $20.5 \%(21 / 102)$ in the present study. 
Table 1

Clinical characteristics of patients with non-HIV PcP

\begin{tabular}{|l|l|}
\hline Variables & $\mathrm{n}=102$ \\
\hline Age, years & $69.5(22-88)$ \\
\hline Gender, male & $55(53.9)$ \\
\hline Body mass index, $\mathrm{kg} / \mathrm{m}^{2}$ & $21.7(12.6-37.7)$ \\
\hline Underlying diseases & \\
\hline Autoimmune diseases & $46(45.1)$ \\
\hline Hematological malignancies & $19(18.6)$ \\
\hline Solid malignancies & $18(17.7)$ \\
\hline Others & $19(18.6)$ \\
\hline Treatment for underlying diseases & $34(33.3)$ \\
\hline Corticosteroids alone & $53(52.0)$ \\
\hline Corticosteroids + other treatments & $15(14.7)$ \\
\hline Others (without corticosteroids) & $15(2-400)$ \\
\hline $\begin{array}{l}\text { Corticosteroid dosage for underlying diseases, mg/day } \\
\text { (Prednisolone equivalent, range) }\end{array}$ & $2(1.9)$ \\
\hline $\begin{array}{l}\text { PcP prophylaxis } \\
\text { number ( }) \text { with percentage (\%). Corticosteroid dosage was expressed as equivalent dose of } \\
\text { prednisolone ( }=78) . \text { Abbreviations: PcP; Pneumocystis pneumonia }\end{array}$ \\
\hline $\begin{array}{l}\text { Outcome from onset of PcP to treatment initiation (range) } \\
\text { 30-day mortality }\end{array}$ & $4.5(1-36)$ \\
\hline
\end{tabular}

\section{Comparison of clinical data between survivors and non- survivors}

In the 30 days following treatment initiation, 81 patients survived and 21 died. The comparison of clinical data between the survivors and non-survivors is shown in Table 2. Non-survivors were significantly older than survivors ( 75 years vs. 67 years, $p=0.019$ ). The prevalence of underlying diseases did not 
significantly differ between the survivors and non-survivors. However, the use of corticosteroids was more frequent in the non-survivors than in the survivors for treatment of their underlying diseases. The corticosteroid dosage administered for therapy of underlying diseases at the onset of PcP was significantly higher in the non-survivors than in the survivors $(25 \mathrm{mg} /$ day vs. $15 \mathrm{mg} / \mathrm{day}, \mathrm{p}=0.001)$. There were no significant differences in PcP prophylaxis between the survivors and non-survivors, and the most frequently used drug for prophylaxis was TMP-SMX, in both survivors and non-survivors. There was no significant difference in the interval of days from the onset of PcP to the treatment initiation between the non-survivors and survivors $(p=0.162)$. Respiratory failure was significantly more prevalent in the nonsurvivors than in the survivors $(95.2 \%$ vs. $60.4 \%, p=0.002)$ at treatment initiation.

\section{Comparison of laboratory findings before treatment between survivors and non-survivors}

The comparison of the laboratory findings before treatment showed that the non-survivors presented with significantly higher levels of $A-a_{2} O_{2}$ (194.6 Torr vs. 64.3 Torr, $\left.p=0.001\right)$, LDH (525 IU/L vs. 374 IU/L, $p=$ $0.001)$, and BUN (31.5 mg/dL vs. $20.0 \mathrm{mg} / \mathrm{dL}, \mathrm{p}=0.004)$, as well as a higher BUN/Alb ratio (16.0 vs. 7.1, $\mathrm{p}=0.002$ ) than the survivors (Table 2). In addition, the non-survivors showed significantly lower $\mathrm{PaO}_{2}$ to fraction of inspired oxygen $\left(\mathrm{FiO}_{2}\right)$ ratio (166.8 vs. 251.9, $\left.\mathrm{p}=0.023\right)$ and levels of total protein (TP) $(5 \mathrm{~g} / \mathrm{dL}$ vs. $5.6 \mathrm{~g} / \mathrm{dL}, \mathrm{p}=0.033)$ and serum Alb $(2.2 \mathrm{~g} / \mathrm{dL}$ vs. $2.8 \mathrm{~g} / \mathrm{dL}, \mathrm{p}=0.002)$ than the survivors (Table 2). There were no significant differences in C-reactive protein, $\mathrm{KL}-6$, and plasma $(1 \rightarrow 3)-\beta$-D-glucan levels between the survivors and non-survivors (Table 2).

\section{Treatments}

TMP-SMX was the most frequently used anti-PcP drug in the initial treatment regimen for both survivors and non-survivors. The administration of adjuvant corticosteroid therapy for the treatment of non-HIV PcP was significantly more frequent in the non-survivors than in the survivors $(90.4 \%$ vs. $70.3 \%, p=$ 0.016) (Table 2). The requirement of IPPV was significantly higher among the non-survivors than the survivors $(61.9 \%$ vs. $13.5 \%, \mathrm{p}<0.001$, Table 2$)$. 
Table 2

Comparison of clinical data between survivors and non-survivors

\begin{tabular}{|c|c|c|c|}
\hline Variables & Survivors $(n=81)$ & non-survivors $(n=21)$ & $\begin{array}{l}\mathrm{P} \\
\text { Value }\end{array}$ \\
\hline Age, years (range) & $67(22-88)$ & $75(56-86)$ & 0.019 \\
\hline Gender, male & $42(51.8 \%)$ & $13(65 \%)$ & 0.41 \\
\hline Body mass index, kg/m² (range) & $\begin{array}{l}21.4(12.6-35.0, \mathrm{n} \\
=80)\end{array}$ & $22.3(16.0-37.7)$ & 0.621 \\
\hline \multicolumn{4}{|l|}{ Underlying diseases } \\
\hline Autoimmune diseases & 38 (46.9\%) & $8(38 \%)$ & 0.469 \\
\hline Hematological malignancies & $14(17.2 \%)$ & $5(23.8 \%)$ & 0.344 \\
\hline Solid malignancies & $15(18.5 \%)$ & $3(14.2 \%)$ & 0.464 \\
\hline Others & $14(17.2 \%)$ & $5(23.8 \%)$ & 0.344 \\
\hline \multicolumn{4}{|l|}{ Treatment for underlying diseases } \\
\hline Corticosteroids alone & $24(29.6 \%)$ & $10(47.6 \%)$ & 0.119 \\
\hline Corticosteroids + other treatments & $42(51.8 \%)$ & $11(52.3 \%)$ & 0.966 \\
\hline Others (without corticosteroids) & $15(18.6 \%)$ & $0(0.0 \%)$ & 0.023 \\
\hline $\begin{array}{l}\text { Corticosteroid dosage for underlying } \\
\text { diseases, } \mathrm{mg} / \text { day }\end{array}$ & $15(2-60, n=59)$ & $25(8-400, n=19)$ & 0.001 \\
\hline \multicolumn{4}{|l|}{ (Prednisolone equivalent, range) } \\
\hline PcP prophylaxis & $1(1.2 \%)$ & $1(5 \%)$ & 0.371 \\
\hline $\begin{array}{l}\text { Days from onset of PcP to treatment } \\
\text { initiation (range) }\end{array}$ & $6(1-36)$ & $4(1-19)$ & 0.162 \\
\hline Respiratory failure & $49(60.4 \%)$ & $20(95.2 \%)$ & 0.002 \\
\hline Hypotension & $8(9.9 \%)$ & $2(9.5 \%)$ & 0.631 \\
\hline Disturbance of consciousness & $3(3.7 \%)$ & $6(28.5 \%)$ & 0.002 \\
\hline \multicolumn{4}{|l|}{ Initial Treatment for PcP } \\
\hline TMP/SMX & $78(96.2 \%)$ & $20(95.2 \%)$ & 0.608 \\
\hline Pentamidine & $2(2.4 \%)$ & $1(4.7 \%)$ & 0.503 \\
\hline Atovaquone & $1(1.2 \%)$ & & 0.794 \\
\hline Adjunctive corticosteroid therapy & $57(70.3 \%)$ & $19(90.4 \%)$ & 0.016 \\
\hline
\end{tabular}




\begin{tabular}{|c|c|c|c|}
\hline Variables & Survivors $(n=81)$ & non-survivors $(n=21)$ & $\begin{array}{l}\mathrm{P} \\
\text { Value }\end{array}$ \\
\hline Respiratory support with IPPV & $11(13.5 \%)$ & $13(61.9 \%)$ & $\begin{array}{l}< \\
0.001\end{array}$ \\
\hline A-DROP score (median) & $1(n=79)$ & $3(n=20)$ & $\dot{0} 001$ \\
\hline \multicolumn{4}{|l|}{ A-DROP score } \\
\hline 0 & $11(13.6 \%)$ & $0(0.0 \%)$ & \\
\hline 1 & $30(37.0 \%)$ & $1(4.8 \%)$ & \\
\hline 2 & $19(23.5 \%)$ & $8(38.1 \%)$ & \\
\hline 3 & $14(17.3 \%)$ & $8(38.1 \%)$ & \\
\hline 4 & $5(6.2 \%)$ & $1(4.8 \%)$ & \\
\hline 5 & $0(0.0 \%)$ & $2(9.4 \%)$ & \\
\hline unkown & $2(2.4 \%)$ & $1(4.8 \%)$ & \\
\hline \multicolumn{4}{|c|}{$\begin{array}{l}\text { Laboratory findings before treatment for } \\
\text { PCP }\end{array}$} \\
\hline $\mathrm{PaO}_{2} / \mathrm{FiO}_{2}$ ratio & $251.9(60.1-447.1)$ & $166.8(64.5-405.2)$ & 0.023 \\
\hline $\mathrm{A}-\mathrm{aDO}_{2}$, Torr & $64.3(17.7-548.5)$ & $194.6(19.1-530.6)$ & 0.001 \\
\hline White blood cell, $/ \mu \mathrm{L}$ & $8000(980-19600)$ & $7540(1850-22600)$ & 0.918 \\
\hline Neutrophils, $/ \mu \mathrm{L}$ & $\begin{array}{l}6869(666-18780 \\
\mathrm{n}=80)\end{array}$ & $\begin{array}{l}6484.5(1099-22000 \\
\mathrm{n}=20)\end{array}$ & 0.803 \\
\hline Lymphocytes, $/ \mu \mathrm{L}$ & $\begin{array}{l}655(77-3010, n= \\
80)\end{array}$ & $\begin{array}{l}634.5(40-2530, \mathrm{n}= \\
20)\end{array}$ & 0.823 \\
\hline Total protein, $\mathrm{g} / \mathrm{dL}$ & $\begin{array}{l}5.6(3.8-7.6, n= \\
75)\end{array}$ & $5(3.6-7.7, n=19)$ & 0.033 \\
\hline Alb, g/dL & 2.8) $(1.4-4.1, \mathrm{n}=$ & $2.2(1.6-3.4)$ & 0.002 \\
\hline $\mathrm{LDH}, \mathrm{IU} / \mathrm{L}$ & $374(137-902)$ & $525(269-1395)$ & 0.001 \\
\hline Total bilirubin, $\mathrm{mg} / \mathrm{dL}$ & $\begin{array}{l}0.56(0.18-21.06 \\
\mathrm{n}=80)\end{array}$ & $0.75(0.1-4.5, \mathrm{n}=20)$ & 0.148 \\
\hline $\mathrm{BUN}, \mathrm{mg} / \mathrm{dL}$ & $20(6-148.7)$ & $31.5(12-88)$ & 0.004 \\
\hline Creatinine, $\mathrm{mg} / \mathrm{dL}$ & $0.88(0.36-11.91)$ & $0.83(0.4-7)$ & 0.685 \\
\hline $\mathrm{CRP}, \mathrm{mg} / \mathrm{dL}$ & $7.4(0.1-31.6)$ & $7.74(0.5-26.1)$ & 0.901 \\
\hline
\end{tabular}




\begin{tabular}{|c|c|c|c|}
\hline Variables & Survivors $(n=81)$ & non-survivors $(n=21)$ & $\begin{array}{l}P \\
\text { Value }\end{array}$ \\
\hline $\mathrm{KL}-6, \mathrm{U} / \mathrm{mL}$ & $\begin{array}{l}613(32.1-11109, \mathrm{n} \\
=56)\end{array}$ & $\begin{array}{l}918.8(324-3656, n= \\
15)\end{array}$ & 0.111 \\
\hline$(1 \rightarrow 3)$ - $\beta$-D-glucan, $\mathrm{pg} / \mathrm{mL}$ & $67.6(11.6-912)$ & 99.7 (12.07-9690) & 0.983 \\
\hline BUN/Alb ratio & $\begin{array}{l}7.1 \\
79)\end{array}(1.6-49.5, n=$ & $16(4.4-45.6)$ & 0.002 \\
\hline \multicolumn{4}{|c|}{$\begin{array}{l}\text { Continuous variables are expressed as median (range) and categorical variables are expressed as } \\
\text { number (n) with percentage (\%). Corticosteroid dosage was expressed as equivalent dose of } \\
\text { prednisolone. Abbreviations: } \mathrm{PcP} ; \text { Pneumocystis pneumonia, IPPV; invasive positive pressure } \\
\text { ventilation, TMP/SMX; trimethoprim/sulfamethoxazole, } \mathrm{PaO}_{2} \text {; partial pressure of arterial oxygen, } \mathrm{FiO}_{2} \text {; } \\
\text { fraction of inspired oxygen, } \mathrm{A}-\mathrm{aDO}_{2} \text {; alveolar-arterial oxygen gradient, Alb; albumin, } \mathrm{LDH} \text {; lactate } \\
\text { dehydrogenase, } \mathrm{BUN} \text {; blood urea nitrogen, } \mathrm{CRP} ; \mathrm{C} \text {-reactive protein }\end{array}$} \\
\hline
\end{tabular}

\section{Prognostic factors for non-HIV PcP in relation to 30-day mortality}

Prognostic factors in relation to 30-day mortality were assessed by multiple logistic regression analysis. The independence of each variable with a $p$ value $<0.05$ obtained upon comparison of the data between the survivors and non-survivors was assessed using Spearman's rank correlation coefficient. Treatment factors were excluded from this analysis to enable examination of the prognostic factors at the beginning of treatment. Age, respiratory failure, $\mathrm{PaO}_{2} / \mathrm{FiO}_{2}$ ratio and TP, Alb, and $\mathrm{BUN}$ levels were not included in the multiple logistic regression analysis because there was a strong correlation between these factors, with a Spearman's rank correlation coefficient of over 0.6 . Therefore, the variables of corticosteroid administration and dosage for underlying diseases, disturbance of consciousness, A-DROP score, A$\mathrm{aDO}_{2}, \mathrm{LDH}$ levels, and BUN/Alb ratio were included in the multivariate logistic regression analysis. Multiple logistic regression analysis revealed that the A-DROP score (odds ratio (OR) 0.530; $95 \%$ confidence interval $(\mathrm{Cl}) 0.307-0.915 ; \mathrm{p}=0.023)$ and BUN/Alb ratio (OR 0.938; 95\% $\mathrm{Cl} 0.893-0.986 ; \mathrm{p}=$ 0.012 ) were independently associated with 30 -day mortality (Table 3 ).

Table 3

Prognostic factors for 30-day mortality for non-HIV PcP

\begin{tabular}{|llll|}
\hline Variable & OR & $95 \%$ Cl & P value \\
\hline A-DROP score & 0.53 & $0.307-0.915$ & 0.023 \\
\hline BUN/Alb ratio & 0.938 & $0.893-0.986$ & 0.012 \\
\hline $\begin{array}{l}\text { Multivariate analysis was performed using multiple logistic regression analysis. Abbreviations: HIV; } \\
\text { human immunodeficiency virus, PcP; Pneumocystis pneumonia, BUN; blood urea nitrogen, Alb; } \\
\text { albumin, OR; odds ratio, Cl; confidence interval }\end{array}$ & \\
\hline
\end{tabular}




\section{Relationship between A-DROP and 30-day mortality}

A-DROP was used for estimating the disease severity of patients at treatment initiation. The median ADROP score was significantly higher in the non-survivors than in the survivors ( 3 vs. $1, p<0.001$, Table 2 ). The 30-day mortality rate was $0 \%$ in the patients with an A-DROP score of $0,3.2 \%$ in those with a score of $1,29.6 \%$ in those with a score of $2,36.6 \%$ in those with a score of 3 , and $37.5 \%$ in those with a score of 4 or 5 (Table 4). There was a positive correlation between 30-day mortality rate and A-DROP score, with the mortality rate rising with an increase in the A-DROP score. Among the various components that form the A-DROP criteria, (age, BUN $\geq 21 \mathrm{mg} / \mathrm{dL}$ or dehydration, respiratory failure, orientation, and hypotension), respiratory failure (OR 6.62; 95\% $\mathrm{Cl} 1.61-27.17 ; \mathrm{p}=0.009)$ and disturbance of consciousness (OR 16.6; $95 \% \mathrm{Cl} 2.22-124.87 ; \mathrm{p}=0.006$ ) were significantly independently associated with 30 -day mortality by multiple logistic regression analysis (Table 5).

Table 4

The 30-day mortality rate classified by A-DROP score

\begin{tabular}{|lll|}
\hline A-DROP score & Number of patients (\%) & 30-day mortality (\%) \\
\hline 0 & $11(11.1 \%)$ & $0(0 \%)$ \\
\hline 1 & $31(31.3 \%)$ & $1(3.2 \%)$ \\
\hline 3 & $27(27.3 \%)$ & $8(29.6 \%)$ \\
\hline 4 or 5 & $22(22.2 \%)$ & $8(36.3 \%)$ \\
\hline Data are presented as number $(\%)$ & $3(37.5 \%)$ \\
\hline
\end{tabular}

Table 5

Determination of prognostic factors for 30-day mortality with the components of A-DROP

\begin{tabular}{|lccc|}
\hline Variable & OR & $95 \% \mathrm{Cl}$ & P value \\
\hline Respiratory failure & 6.62 & $1.61-27.17$ & 0.009 \\
\hline Disturbance of consciousness & 16.6 & $2.22-124.87$ & 0.006 \\
\hline $\begin{array}{l}\text { Multivariate analysis was performed using multiple logistic regression analysis. Components of A- } \\
\text { DROP include age, BUN } \geqq 21 \mathrm{mg} / \mathrm{dL} \text { or dehydration, respiratory failure, disturbance of consciousness, } \\
\text { and hypotension. Abbreviations: OR; odds ratio; Cl, confidence interval, BUN; blood urea nitrogen }\end{array}$ \\
\hline
\end{tabular}

\section{Ability of prognostic factors to estimate 30 -day mortality}


ROC curves were created for evaluating the ability of prognostic factors to estimate 30-day mortality in patients with non-HIV PCP (Figs. 1-4). The area under the curve (AUC) was 0.719 for BUN/Alb ratio (95\% Cl 0.594-0.844), 0.702 for BUN level ( $95 \% \mathrm{Cl} \mathrm{0.577-0.826),} 0.715$ for Alb level (95\% Cl 0.598-0.833), and 0.760 for the A-DROP score (95\% $\mathrm{Cl} 0.659-0.860)$. The cutoff levels were 9.50 (sensitivity, $71.4 \%$; specificity, 65.8\%) for BUN/Alb ratio and 1.50 (sensitivity, 95.0\%; specificity, 51.9\%) for the A-DROP score for the estimation of prognosis of 30-day mortality in the patients with non-HIV PcP.

\section{Discussion}

Previous studies have shown that the prognosis of patients with non-HIV PcP is worse than that of patients with HIV PcP $[5,6]$. The mortality rate associated with non-HIV PcP ranged between $19.6 \%$ and $60 \%$ in previous studies $[5,6]$, while the 30 -day mortality rate was $20.5 \%$ in the present study. The remarkable finding of this study was that the A-DROP score and BUN/Alb ratio were reliable prognostic factors that can be used for estimating the 30-day mortality in patients with non-HIV PcP.

A previous study reported that an increase in PSI score was associated with mortality in non-HIV PCP [7]. It has been shown that the A-DROP score was not significantly different between the non-HIV PcP survivors and non-survivors at admission, but it was significantly higher in the non-survivors than in the survivors at treatment initiation [20]. Consistently, in the present study, the A-DROP score was found to be significantly higher in the non-survivors than in the survivors at treatment initiation ( 3 vs. $1, p<0.001$ ). The 30-day mortality rate was $0 \%$ in those with an A-DROP score of $0,3.2 \%$ in those with a score of 1 , $29.6 \%$ in those with a score $2,36.6 \%$ in those with a score of 3 , and $37.5 \%$ in those with a score of 4 or 5 . Although it was reported that the A-DROP score underestimated the severity of patients with non-HIV PcP, which developed as CAP [20,22], the 30-day mortality rate increased with increased A-DROP score at treatment initiation in the present study. Therefore, A-DROP might be useful for classifying the severity of patients with non-HIV PcP, especially the A-DROP score at treatment initiation for PcP. Previous studies have shown that the 30 -day mortality rate was $0 \%$ for patients with CAP with an A-DROP score of $0,0-$ $3.1 \%$ in those with a score of $1,3.1 \%-4.6 \%$ in those with a score of $2,9.9-15.9 \%$ in those with a score of 3 , and $19.6 \%-34.0 \%$ in those with a score of 4 or 5 [23-25]. Although it might not be accurate to compare our results with the 30-day mortality rate of CAP, the 30-day mortality in those patients with an A-DROP score of 2 or 3 was relatively higher in non-HIV PcP than in CAP, and the 30-day mortality rate in patients with an A-DROP score of 4 or 5 was relatively the same in non-HIV PcP and CAP. The components of the A-DROP score, such as dehydration, disturbance of consciousness, and hypotension, are also the components of the PSI and CURB65, which is a prognostic indicator estimated by five factors (confusion, urea, respiratory rate, blood pressure, age) and developed by the British Thoracic Society [26]. Dehydration, respiratory failure, disturbance of consciousness, and hypotension were indicative of an overall poor condition in patients. The multivariate analysis regarding the relationship between the ADROP components (age, dehydration, respiratory failure, disturbance of consciousness, hypotension) and 30-day mortality demonstrated that respiratory failure and disturbance of consciousness were significantly independently associated with the 30-day mortality of non-HIV PcP patients in the present 
study. Patients with non-HIV PcP and an A-DROP score of $\geq 2$, or those with respiratory failure or disturbance of consciousness at treatment initiation, usually demonstrate poor prognosis.

Patients with pneumonia are often dehydrated, resulting in increased reabsorption of urea by the kidneys. Thus, an increase in BUN levels is frequently observed in patients with pneumonia [27]. Patients with CAP and increased BUN levels had higher mortality rates $[13,16]$. Increased BUN levels are indicative of renal hypoperfusion and thus help to predict the severity of pneumonia [28]. Increased BUN levels were also found to be a poor prognostic factor in patients with non-HIV PCP [8].

Alb is synthesized only in the liver, and the rate of Alb synthesis varies with nutritional status [29]. The rate of Alb synthesis changes markedly in severe illness, and a sustained inflammatory response may lead to long-term inhibition of Alb synthesis [29]. Serum Alb levels are reduced during acute and chronic inflammatory processes because of inhibition of Alb synthesis in the liver [30]. Hypoalbuminemia is thought to reflect systemic inflammatory reactions and malnutrition. Hypoalbuminemia in patients with non-HIV PcP might be associated with acute inflammation caused by PcP and chronic inflammation caused by underlying diseases. Low serum Alb levels reduce plasma colloid osmotic pressure, thereby increasing pulmonary vascular permeability [31-33]. Hypoalbuminemia was reported to be associated with increased pulmonary vascular permeability and lung injury in critically ill patients [34]. Thus, taking all these points into consideration, hypoalbuminemia can be seen as being associated with poor prognosis in patients with non-HIV PcP, by the same mechanism.

Both high BUN and low serum Alb levels were poor prognostic factors in patients with non-HIV PcP $[8,10]$. This study further revealed that the non-survivors had significantly higher levels of BUN and lower levels of serum Alb than those in the survivors. In addition, high BUN and low serum Alb levels were poor prognostic factors for patients with CAP [13-16], and the BUN/Alb ratio was useful in predicting the mortality of patients with CAP $[19,35]$. Moreover, the BUN/Alb ratio was also a useful marker for predicting the mortality in patients with HAP and aspiration pneumonia $[17,18]$. These findings are consistent with the present study, which revealed that the increased BUN/Alb ratio before treatment was significantly associated with 30-day mortality in patients with non-HIV PcP. Furthermore, the AUC of the ROC curve was 0.719 for BUN/Alb ratio (95\% Cl 0.594-0.844), with a value of 0.702 for BUN $(95 \% \mathrm{Cl}$ $0.577-0.826)$ and 0.715 for Alb (95\% Cl 0.598-0.833). The BUN/Alb ratio seems superior to either BUN or serum Alb alone in predicting prognosis in patients with non-HIV PcP. BUN and serum Alb are commonly measured biochemical markers in patients with non-HIV PcP; therefore, the BUN/Alb ratio is a convenient and useful marker for predicting the prognosis of these.

This study has several limitations. Some clinical data were missing in this retrospective study. Data regarding the status of disturbance of consciousness and dehydration might be inaccurate because these factors were evaluated on the basis of medical records. In addition, the evaluation of dehydration and disturbance of consciousness were dependent on the subjective observation of the physician, and the reported A-DROP scores might differ among physicians. 


\section{Conclusions}

In conclusion, a high A-DROP score and BUN/Alb ratio were independent prognostic predictors for 30-day mortality in patients with non-HIV PcP. BUN and serum Alb levels are commonly examined in the clinical sittings in patients with non-HIV PcP. Thus, the BUN/Alb ratio can be a convenient and useful marker for predicting prognosis in these patients. However, more attention needs to be focused on the treatment strategies for patients with non-HIV PCP presenting a high A-DROP score and/or BUN/Alb ratio because of the high risk for poor prognosis. Further prospective studies are needed to confirm the findings of this study.

\section{Abbreviations}

A- $\mathrm{ADO}_{2}$ : alveolar-arterial oxygen gradient; $\mathrm{A}-\mathrm{DROP}$ : age, dehydration, respiratory failure, orientation, systolic blood pressure; Alb: albumin; AUC: area under the curve; BUN: blood urea nitrogen; BUN/Alb: blood urea nitrogen to serum albumin (BUN/Alb); CAP: community-acquired pneumonia; Cl: confidence interval; $\mathrm{FiO}_{2}$ : fraction of inspired oxygen; GCS: Glasgow Coma Scale; HAP: hospital-acquired pneumonia; HIV: human immunodeficiency virus; IPPV: invasive positive-pressure ventilation; JRS: Japanese Respiratory Society; LDH: lactate dehydrogenase; OR: odds ratio; $\mathrm{PaO}_{2}$ : partial pressure of arterial oxygen; PcP: Pneumocystis pneumonia; PSI: pneumonia severity index; ROC: receiver-operating characteristic; $\mathrm{SpO}_{2}$ : percutaneous oxygen saturation; TMP/SMX: trimethoprim/sulfamethoxazole; TP: total protein

\section{Declarations}

\section{Ethics approval and consent to participate}

The present study was approved by the Ethics Committees of Shinshu University (approval number 4213). The authors assert that this work has been carried out in accordance with the Code of Ethics of the World Medical Association (Declaration of Helsinki) for experiments involving humans. Under common law and ethics, obtaining informed patient consent was not required for the methods used in this study. This study used an opt-out consent model, and patients could choose to opt out at any time and have their data removed from the registry.

\section{Consent for publication}

Not applicable.

\section{Availability of data and materials}

Not applicable.

\section{Competing interests}

The authors declare that they have no competing interests. 


\section{Funding}

This research did not receive any specific grant from funding agencies in the public, commercial, or notfor-profit sectors.

\section{Authors' contributions}

JA and $A U$ designed the study. JA, AU, MK, AM, TH, FY, and SK collected the data. JA, AU, MK, YI, and MH analyzed and interpreted the data. JA and AU wrote the manuscript. All authors reviewed the manuscript.

\section{Acknowledgements}

The authors would like to thank Dr Y. Droma (First Department of Internal Medicine at Shinshu University School of Medicine in Matsumoto City, Nagano, Japan) for advice and expertise.

\section{References}

1. Kelley CF, Checkley W, Mannino DM, Franco-Paredes C, Del Rio C, Holguin F. Trends in hospitalizations for AIDS-associated Pneumocystis jirovecii Pneumonia in the United States (1986 to 2005). Chest. 2009;136:190-7. doi:10.1378/chest.08-2859.

2. Mansharamani NG, Garland R, Delaney D, Koziel H. Management and outcome patterns for adult Pneumocystis carinii pneumonia, 1985 to 1995: comparison of HIV-associated cases to other immunocompromised states. Chest. 2000;118:704-11. doi:10.1378/chest.118.3.704.

3. Arend SM, Kroon FP, van't Wout JW. Pneumocystis carinii pneumonia in patients without AIDS, 1980 through 1993. An analysis of 78 cases. Arch Intern Med. 1995;155:2436-41.

doi:10.1001/archinte.1995.00430220094010.

4. Maini R, Henderson KL, Sheridan EA, Lamagni T, Nichols G, Delpech V, et al. Increasing Pneumocystis pneumonia, England, UK, 2000-2010. Emerg Infect Dis. 2013;19:386-92. doi:10.3201/eid1903.121151.

5. Bollée G, Sarfati C, Thiéry G, Bergeron A, de Mirands S, Menotti J, et al. Clinical picture of Pneumocystis jiroveci pneumonia in cancer patients. Chest. 2007;132:1305-10. doi:10.1378/chest.07-0223.

6. Thomas CF Jr, Limper AH. Pneumocystis pneumonia. N Engl J Med. 2004;350:2487-98.

7. Hardak E, Neuberger A, Yigla M, Berger G, Finkelstein R, Sprecher H, et al. Outcome of Pneumocystis jirovecii pneumonia diagnosed by polymerase chain reaction in patients without human immunodeficiency virus infection. Respirology. 2012;17:681-6. doi:10.1111/j.14401843.2012.02158.x.

8. Kim SJ, Lee J, Cho YJ, Park YS, Lee, C-H, Yoon, HI, et al. Prognostic factors of Pneumocystis jirovecii pneumonia in patients without HIV infection. J Infect. 2014;69:88-95. doi:10.1016/j.jinf.2014.02.015. 
9. Ko Y, Jeong BH, Park HY, Koh, W-J, Suh GY, Chung MP, et al. Outcomes of Pneumocystis pneumonia with respiratory failure in HIV-negative patients. J Crit Care. 2014;29:356-61. doi:10.1016/j.jcrc.2013.12.005.

10. Yasufumi M, Yuichiro S, Yoshitsugu I, Yamamoto M, Shirano M, Matsushima A, et al. Clinical characteristics of Pneumocystis pneumonia in non-HIV patients and prognostic factors including microbiological genotypes. BMC Infect Dis. 2011;11:76. doi:10.1186/1471-2334-11-76.

11. Fine MJ, Auble TE, Yealy DM, Hanusa BH, Weissfeld LA, Singer DE, et al. A prediction rule to identify low-risk patients with community-acquired pneumonia. N Engl J Med. 1997;336:243-50. doi:10.1056/nejm199701233360402.

12. Miyashita N, Matsushima T, Oka M; Japanese Respiratory Society. The JRS guidelines for the management of community-acquired pneumonia in adults: an update and new recommendations. Intern Med. 2006;45:419-28. doi:10.2169/internalmedicine.45.1691.

13. Farr BM, Sloman AJ, Fisch MJ. Predicting death in patients hospitalized for community-acquired pneumonia. Ann Intern Med. 1991;115:428-36. doi:10.7326/0003-4819-115-6-428.

14. Lee JH, Kim J, Kim K, Jo, YH, Rhee JE, Kim TY, et al. Albumin and C-reactive protein have prognostic significance in patients with community-acquired pneumonia. J Crit Care. 2011;26:287-94. doi:10.1016/j.jcrc.2010.10.007.

15. Potgieter PD, Hammond JM. The intensive care management, mortality and prognostic indicators in severe community-acquired pneumococcal pneumonia. Intensive Care Med. 1996;22:1301-6. doi:10.1007/bf01709542.

16. Raz R, Dyachenko P, Levy Y, Flatau E, Reichman N. A predictive model for the management of community-acquired pneumonia. Infection. 2003;31:3-8. doi:10.1007/s15010-002-2083-4.

17. Feng DY, Zhou YQ, Zou XL, Zhou M, Yang H-L, et al. Elevated blood urea nitrogen-to-serum albumin ratio as a factor that negatively affects the mortality of patients with hospital-acquired pneumonia. Can J Infect Dis Med Microbiol. 2019;2019:1547405. doi:10.1155/2019/1547405.

18. Ryu S, Oh SK, Cho SU, You Y, Park JS, Min JH, et al. Utility of the blood urea nitrogen to serum albumin ratio as a prognostic factor of mortality in aspiration pneumonia patients. Am J Emerg Med. 2020;S0735-6757(20)30118-2. doi:10.1016/j.ajem.2020.02.045.

19. Ugajin M, Yamaki K, Iwamura N, Yagi T, Asano T. Blood urea nitrogen to serum albumin ratio independently predicts mortality and severity of community-acquired pneumonia. Int J Gen Med. 2012;5:583-9. doi:10.2147/ijgm.s33628.

20. Asai N, Motojima S, Ohkuni Y, Matsunuma R, Nakasima K, Iwasaki T, et al. Early diagnosis and treatment are crucial for the survival of Pneumocystis pneumonia patients without human immunodeficiency virus infection. J Infect Chemother. 2012a;18:898-905. doi:10.1007/s10156-0120441-4.

21. Kosaka M, Ushiki A, Ikuyama Y, Hirai K, Matsuo A, Hachiya T, et al. A four-center retrospective study of the efficacy and toxicity of low-dose trimethoprim-sulfamethoxazole for the treatment of 
Pneumocystis pneumonia in patients without HIV infection. Antimicrob Agents Chemother. 2017;61:e01173-17. doi:10.1128/aac.01173-17.

22. Asai N, Motojima S, Ohkuni Y, Matsunuma R, Nakasima K, Iwasaki T, et al. Non-HIV Pneumocystis pneumonia: do conventional community-acquired pneumonia guidelines underestimate its severity? Multidiscip Respir Med. 2012b;7:2. doi:10.1186/2049-6958-7-2.

23. Kohno S, Seki M, Watanabe A; CAP Study Group. Evaluation of an assessment system for the JRS 2005: A-DROP for the management of CAP in adults. Intern Med. 2011;50:1183-91. doi:10.2169/internalmedicine.50.4651.

24. Shindo Y, Sato S, Maruyama E, Ohashi T, Ogawa M, Imaizumi K, et al. Comparison of severity scoring systems A-DROP and CURB-65 for community-acquired pneumonia. Respirology. 2008;13:731-5. doi:10.1111/j.1440-1843.2008.01329.x.

25. Usui $K$, Tanaka $Y$, Noda $H$, Ishihara $T$, et al. [Comparison of three prediction rules for prognosis in community acquired pneumonia: Pneumonia Severity Index (PSI), CURB-65, and A-DROP] (Article in Japanese). Nihon Kokyuki Gakkai Zasshi. 2009;47:781-5.

26. Lim WS, van der Eerden MM, Laing R, Boersma WG, Karalus N, Town Gl, et al. Defining community acquired pneumonia severity on presentation to hospital: an international derivation and validation study. Thorax. 2003;58:377-82. doi:10.1136/thorax.58.5.377.

27. Woodford-Williams E. Respiratory tract disease. Diagnosis and management of pneumonia in the aged. Br Med J. 1966;1:467-70. doi:10.1136/bmj.1.5485.467.

28. Evrim E, Derya H, Beyza D, Gulhan M. The role of albumin level and blood urea nitrogen/albumin ratio in prediction of prognosis of community acquired pneumonia. J Pulm Respir Med. 2013;3:5. doi:10.4172/2161-105x.1000159.

29. Nicholson JP, Wolmarans MR, Park GR. The role of albumin in critical illness. Br J Anaesth. 2000;85:599-610.

30. Di Napoli M, Behrouz R, Topel CH, Misra V, Pomero F, Giraud A, et al. Hypoalbuminemia, systemic inflammatory response syndrome, and functional outcome in intracerebral hemorrhage. $\mathrm{J}$ Crit Care. 2017;41:247-53. doi:10.1016/j.jcrc.2017.06.002.

31. Arquès $S$, Ambrosi P, Gélisse R, Luccioni R, Habib, G. Hypoalbuminemia in elderly patients with acute diastolic heart failure. J Am Coll Cardiol. 2003;42:712-6. doi:10.1016/s0735-1097(03)00758-7.

32. Lum H, Siflinger-Birnboim A, Blumenstock F, Malik AB. Serum albumin decreases transendothelial permeability to macromolecules. Microvasc Res. 1991;42:91-102. doi:10.1016/0026-2862(91)900770.

33. Rackow EC, Fein IA, Leppo J. Colloid osmotic pressure as a prognostic indicator of pulmonary edema and mortality in the critically ill. Chest. 1977;72:709-13. doi:10.1378/chest.72.6.709.

34. Aman J, van der Heijden M, van Lingen A, Girbes ARJ, van Nieuw Amerongen GP, van Hinsbergh VWM, et al. Plasma protein levels are markers of pulmonary vascular permeability and degree of lung injury in critically ill patients with or at risk for acute lung injury/acute respiratory distress syndrome. Crit Care Med. 2011;39:89-97. doi:10.1097/ccm.0b013e3181feb46a. 
35. Tokgoz Akyil F, Yalcinsoy M, Hazar A, Cilli A, Celenk B, Kilic O, et al. Prognosis of hospitalized patients with community-acquired pneumonia. Pulmonology. 2018;S2173-5115(17)30156-2. doi:10.1016/j.rppnen.2017.07.010.

\section{Figures}

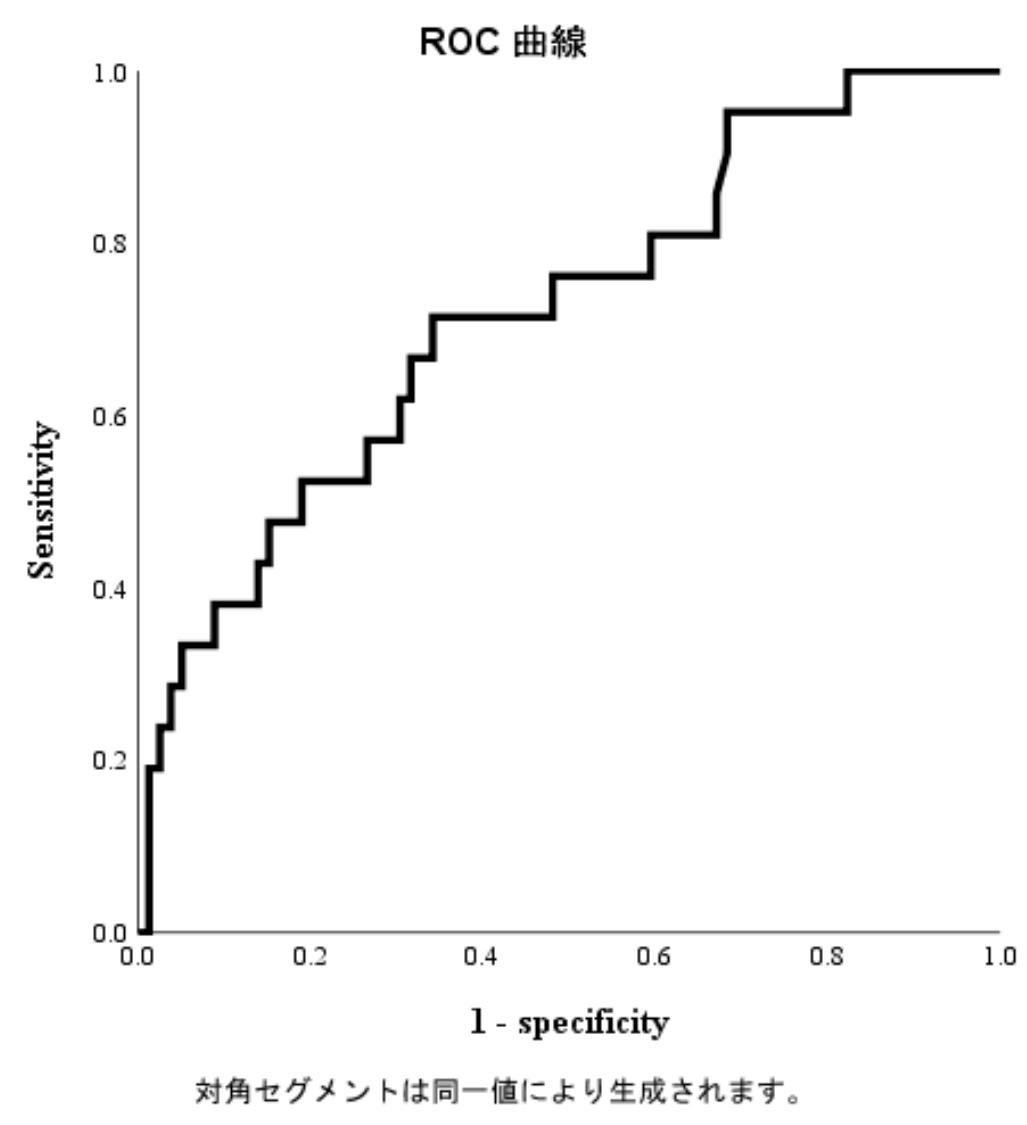

Figure 1

ROC curve of BUN/Alb ratio for predicting the 30-day mortality for non-HIV PCP. The AUC was 0.719 (95\% Cl 0.594-0.844). Abbreviations: ROC; Receiver-operating characteristics, BUN; blood urea nitrogen, Alb; albumin, HIV; human immunodeficiency virus, PcP, Pneumocystis pneumonia; AUC; area under the curve, $\mathrm{Cl}$; confidence interval 


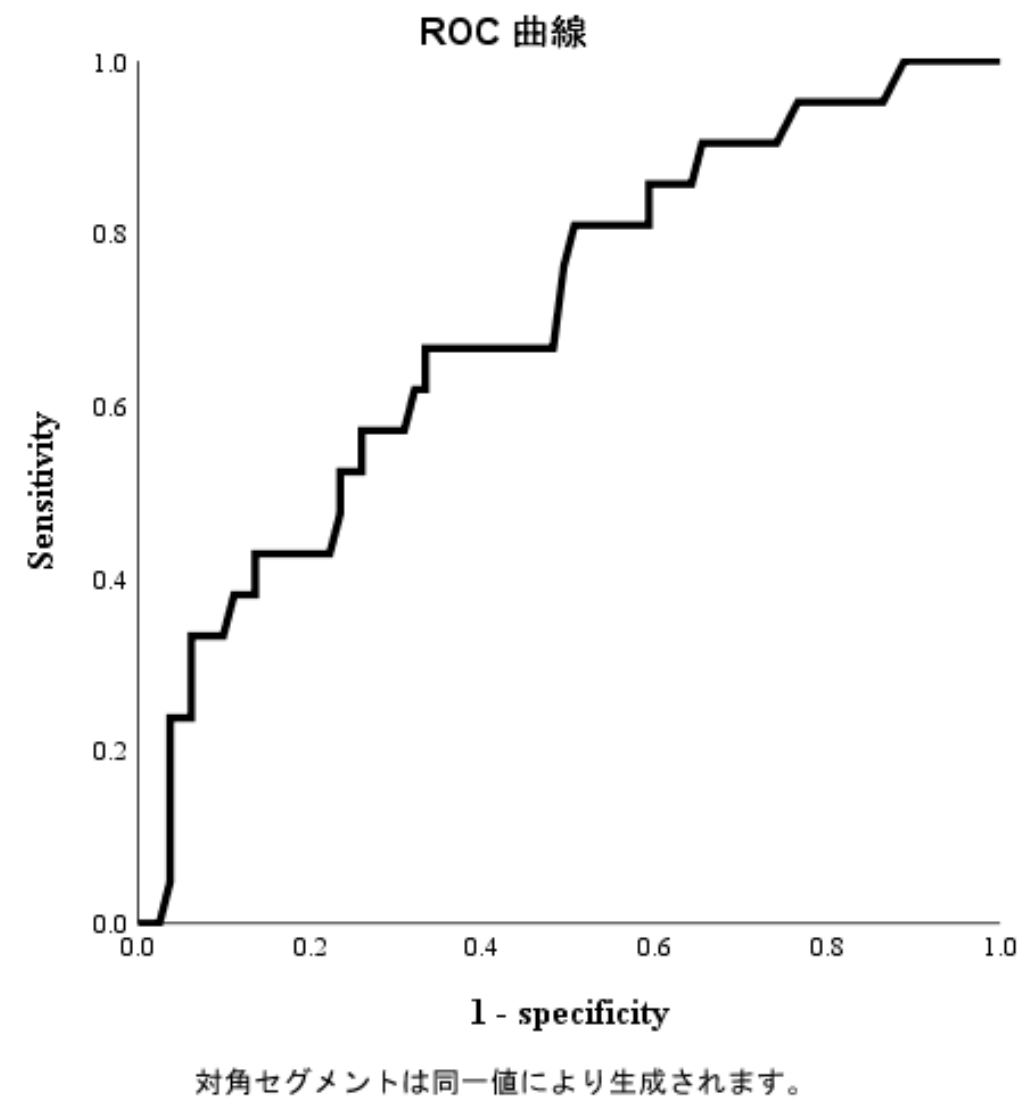

\section{Figure 2}

ROC curve of BUN for predicting the 30-day mortality for non-HIV PCP. The AUC was $0.702(95 \% \mathrm{Cl} 0.577-$ 0.826). Abbreviations: ROC; Receiver-operating characteristics, BUN; blood urea nitrogen, HIV; human immunodeficiency virus, PcP; Pneumocystis pneumonia, AUC; area under the curve, Cl; confidence interval 


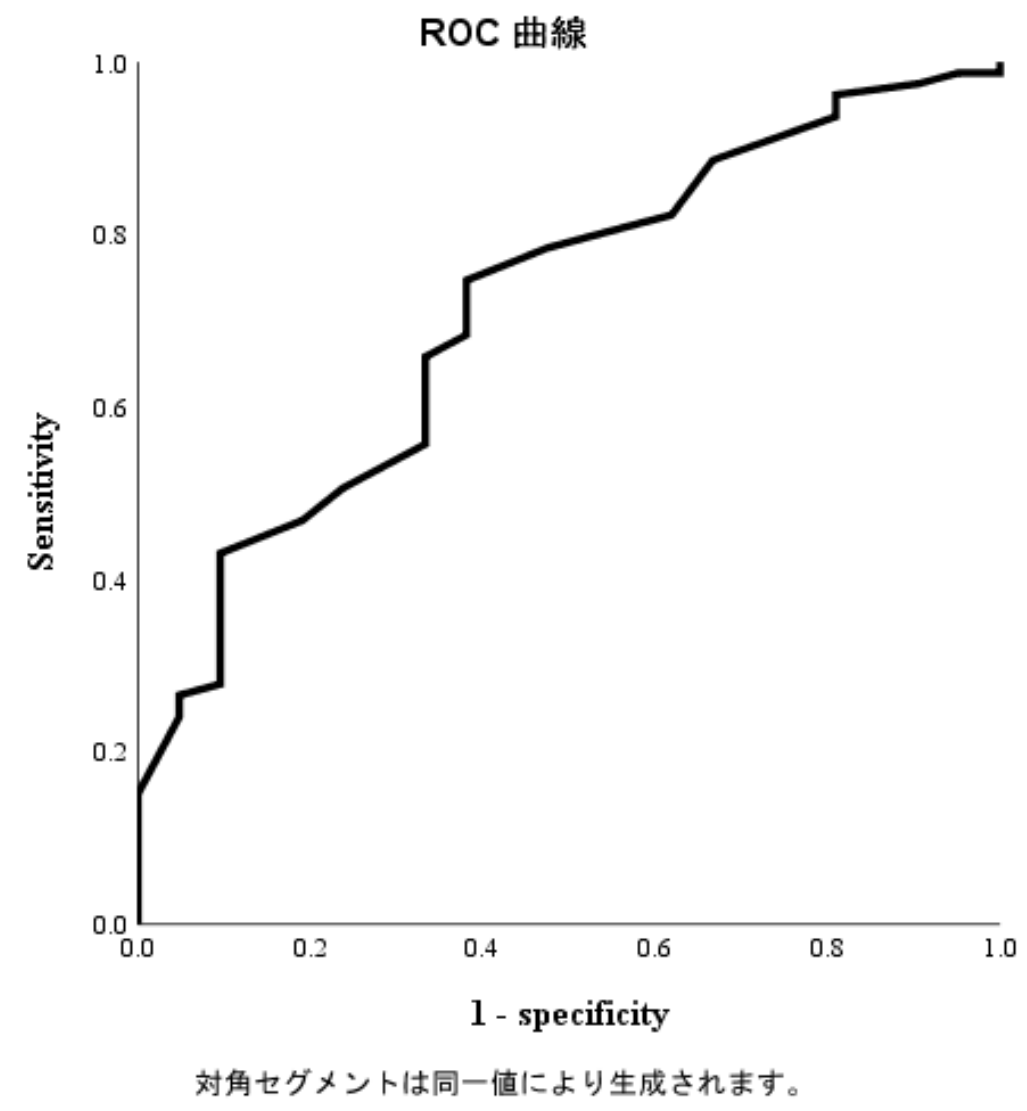

\section{Figure 3}

ROC curve of Alb for predicting the 30-day mortality for non-HIV PcP. The AUC was $0.715(95 \% \mathrm{Cl} 0.598-$ 0.833). Abbreviations: ROC; Receiver-operating characteristics, Alb; albumin, HIV; human immunodeficiency virus, PcP; Pneumocystis pneumonia, AUC; area under the curve, Cl; confidence interval 


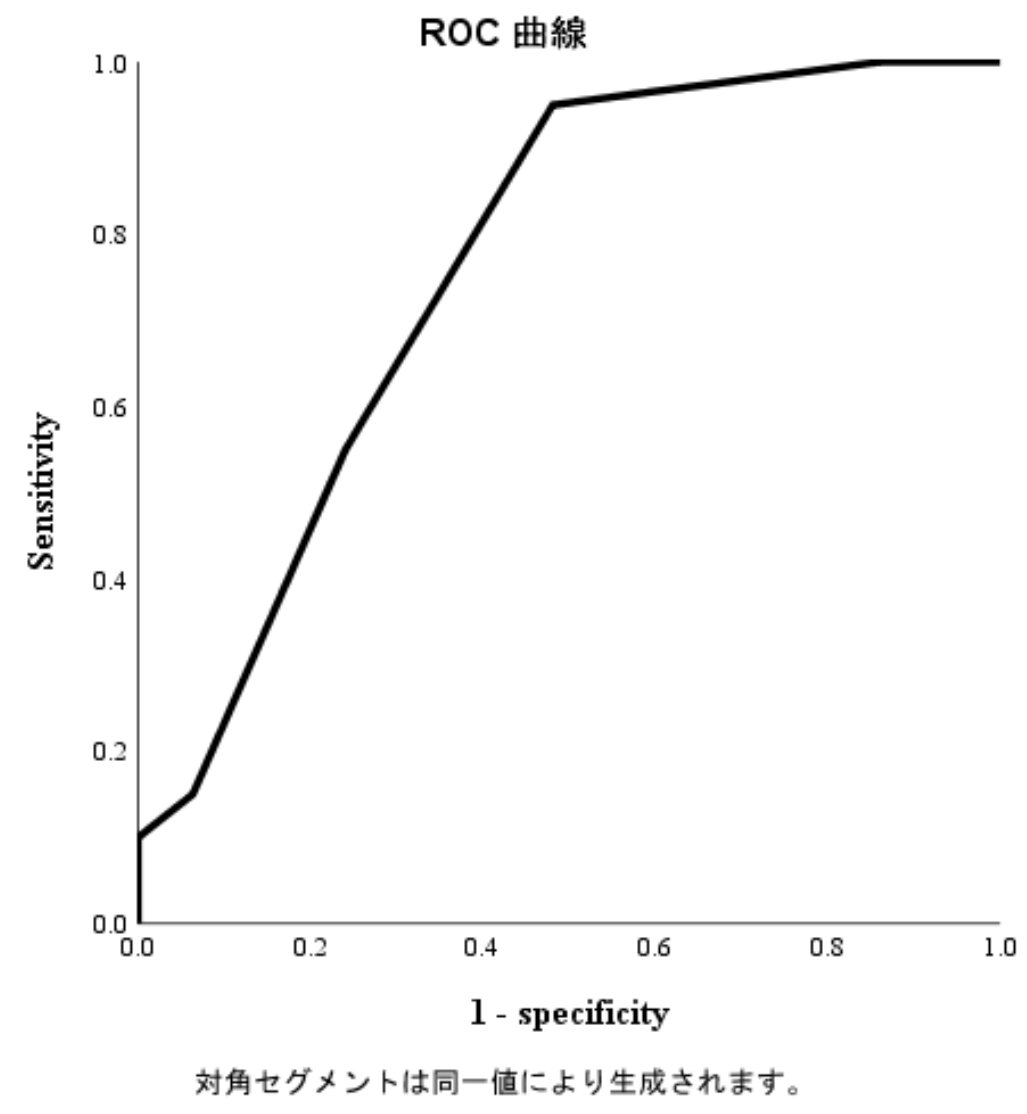

\section{Figure 4}

ROC curve of A-DROP for predicting the 30-day mortality for non-HIV PCP. The AUC was $0.76(95 \% \mathrm{CI}$ 0.659-0.860). Abbreviations: ROC; Receiver-operating characteristics, HIV; human immunodeficiency virus, PcP; Pneumocystis pneumonia, AUC; area under the curve, $\mathrm{Cl}$; confidence interval 